\title{
Nouveau partenaire Teva Pharma SA - Acheter ensemble - profiter ensemble
}

Nous sommes heureux de vous présenter le nouveau partenariat de collaboration avec la société Teva Pharma SA.

Teva est synonyme de génériques. Et bien plus encore! En effet, avec sa philosophie de fournir toujours plus elle s'est hissée au rang d'entreprise pharmaceutique active au plan international qui a plus d'une corde à son arc. Dans son propre laboratoire de recherche, Teva développe avec succès des médicaments innovants ainsi que des principes actifs novateurs. Parce que son but est non seulement d'œuvrer pour préserver la santé, mais de maintenir également les coûts à un niveau abordable, la société occupe désormais une position stratégique de leader mondial sur le marché international des génériques. Grâce à ces compétences, Teva est pour vous un partenaire fiable sur le marché pharmaceutique suisse. La société Teva s'efforce d'offrir toujours davantage: plus de service, plus d'engagement et plus de sécurité et de qualité, éléments qu'elle est à même de contrôler de manière optimale dans ses propres établissements de production. Pour obtenir davantage d'informations sur Teva tapez www.tevapharma.ch.

En votre qualité de sociétaire des FMH Services, vous avez la possibilité de faire décompter vos commandes futures passées à Teva Pharma SA directement par l'intermédiaire d'un système de facturation collective. Ainsi, vous n'économiserez pas seulement des frais et des charges administratives, mais vous profiterez aussi d'offres spéciales et exclusives sous forme de rabais.

Vos avantages:

- $\quad 7 \%$ de rabais sur tous les achats effectués auprès de la société Teva Pharma SA pour les nouveaux clients. Si vous faites décompter vos achats pour la première fois par l'intermédiaire de la société FMH Consulting Services, vous profiterez durant la première année d'un rabais de $7 \%$. A partir de la $2^{\mathrm{e}}$ année, le rabais est de $5 \%$.

- Facture mensuelle unique pour tous les achats effectués auprès des fournisseurs contractuels pendant les dernières 4 à 6 semaines.

Avons-nous suscité votre curiosité? Si vous souhaitez vous connecter à notre système de facturation collective, nous vous prions de bien vouloir nous retourner le talon-réponse. Vous recevrez le bulletin de commande par e-mail ou fax et vous pourrez alors passer vos commandes directement au fournisseur. De notre part vous recevrez une facture mensuelle.

Nous sommes persuadés que la collaboration avec ce nouveau partenaire constitue pour vous une bonne prestation de service.

\section{Talon d'inscription facture collective}

\section{Fax 0419210586 ou courriel: mail@fmhservices.ch}

Afin de tirer profit de manière optimale de cette offre spéciale de la société FMH Consulting Services, je souhaite dénouer mes achats futurs auprès de la TEVA Pharma SA par l'intermédiaire du système de facturation collective:

J'ai pris connaissance du fait que la prestation de service «facture collective» de la société FMH Consulting Services est gratuite pour moi.

Nom:

Rue:

Tél. privé:

Numéro RCC:

(anciennement $\mathrm{N}^{\circ}$ de concordat)
Prénom:

NPA/localité:

Tél. prof::

Courriel:

Signature:

Lieu/date: 